\title{
The retinoblastoma tumor suppressor modifies the therapeutic response of breast cancer
}

\author{
Emily E. Bosco, ${ }^{1}$ Ying Wang, ${ }^{1}$ Huan Xu, ${ }^{2}$ Jack T. Zilfou, ${ }^{3}$ Karen E. Knudsen, ${ }^{1}$ \\ Bruce J. Aronow, ${ }^{2}$ Scott W. Lowe, ${ }^{3,4}$ and Erik S. Knudsen ${ }^{1}$ \\ 1Department of Cell Biology, The Vontz Center for Molecular Studies, University of Cincinnati, College of Medicine, Cincinnati, Ohio, USA. \\ ${ }^{2}$ Cincinnati Children's Hospital Medical Center, Cincinnati, Ohio, USA. ${ }^{3}$ Cold Spring Harbor Laboratory, Cold Spring Harbor, New York, USA. \\ ${ }^{4}$ Howard Hughes Medical Institute, Cold Spring Harbor, New York, USA.
}

\begin{abstract}
The retinoblastoma tumor suppressor $(\mathrm{RB})$ protein is functionally inactivated in the majority of human cancers and is aberrant in one-third of all breast cancers. $R B$ regulates $G_{1} / S$-phase cell-cycle progression and is a critical mediator of antiproliferative signaling. Here the specific impact of RB deficiency on E2F-regulated gene expression, tumorigenic proliferation, and the response to 2 distinct lines of therapy was investigated in breast cancer cells. RB knockdown resulted in RB/E2F target gene deregulation and accelerated tumorigenic proliferation, thereby demonstrating that even in the context of a complex tumor cell genome, $R B$ status exerts significant control over proliferation. Furthermore, the RB deficiency compromised the short-term cell-cycle inhibition following cisplatin, ionizing radiation, and antiestrogen therapy. In the context of DNA-damaging agents, this bypass resulted in increased sensitivity to these agents in cell culture and xenograft models. In contrast, the bypass of antiestrogen signaling resulted in continued proliferation and xenograft tumor growth in the presence of tamoxifen. These effects of aberrations in RB function were recapitulated by ectopic E2F expression, indicating that control of downstream target genes was an important determinant of the observed responses. Specific analyses of an RB gene expression signature in 60 human patients indicated that deregulation of this pathway was associated with early recurrence following tamoxifen monotherapy. Thus, because the $\mathrm{RB}$ pathway is a critical determinant of tumorigenic proliferation and differential therapeutic response, it may represent a critical basis for directing therapy in the treatment of breast cancer.
\end{abstract}

\section{Introduction}

Breast cancer is the leading noncutaneous cancer diagnosis in American women, affecting more than 240,000 new patients per year. Treatment options for breast cancer are governed by the estrogen dependence of the tumor cells. Two-thirds of all breast cancers are estrogen receptor (ER) positive, and in these tumors ER serves as a molecular target for hormone ablation therapy (1). Antiestrogens, such as the widely used tamoxifen (Tam), are the first-line therapy for ER-positive tumors and efficiently elicit a $G_{0} / G_{1}$-phase arrest in hormone-dependent cancer cells $(2,3)$. This class of drugs is initially effective in curbing the growth of ER-positive tumors; however, many patients whose tumors initially respond to antiestrogen treatment develop cellular resistance to Tam while maintaining ER-positive disease (4-6). This suggests that genetic lesions downstream of ER bypass the effectiveness of therapy. Second-line therapies for tumors that exhibit resistance to antiestrogens have traditionally included radiation and chemotherapies that function by damaging DNA (e.g., cisplatin). Importantly, the critical determinants for therapeutic response to either antiestrogens or DNA-damaging agents are largely unknown.

Nonstandard abbreviations used: CDDP, cis-diamminedichloroplatinum II; CDK, cyclin-dependent kinase; CDT, charcoal dextran-treated; E2, 17ß-estradiol; ER, estrogen receptor; ICI, ICI 182780; IR, ionizing radiation; MCM7, minichromosome maintenance 7; PCNA, proliferating cell nuclear antigen; RB, retinoblastoma tumor suppressor; shRNA, short hairpin RNA; Tam, tamoxifen.

Conflict of interest: The authors have declared that no conflict of interest exists. Citation for this article: J. Clin. Invest. 117:218-228 (2007). doi:10.1172/JCI28803.
The retinoblastoma tumor suppressor (RB) plays a central role in cell-cycle control and regulates the cellular response to diverse therapeutic agents. In quiescent cells, RB is hypophosphorylated and assembles transcriptional repressor complexes on the promoters of E2F-regulated genes to block cell-cycle progression. In response to mitogenic factors, including estrogen in breast cancer cells, RB is inactivated through hyperphosphorylation catalyzed by the cyclin D-cyclin-dependent kinase 4 (cyclin D-cdk4) and cyclin E-cdk2 complexes (7-9). These modifications are sufficient to disrupt the interaction of RB with E2F proteins, thereby relieving transcriptional repression and permitting cell-cycle progression. In contrast, antimitogenic factors activate RB, inhibiting cell-cycle progression. For example, RB activity is instrumental in the DNA damage-induced cell-cycle checkpoint and is necessary for $\mathrm{G}_{1}$ - and S-phase arrest following DNA-damaging events. Correspondingly, Tam and other antiestrogens function to block RB phosphorylation and engage RB-mediated transcriptional repression of E2F $(2,3,10,11)$.

RB has been reported to be aberrant in approximately $20 \%-35 \%$ of breast cancers $(12,13)$ and has been associated with poor disease outcome. Additionally, loss of heterozygosity or other alterations at the $R b$ locus are routinely observed in primary breast cancer specimens (14-16). Furthermore, the overproduction of cyclin D1 and cyclin $\mathrm{E}$, which mediate the inactivation of $\mathrm{RB}$, are relatively common events in breast cancer (17). Last, microarray analyses have indicated that deregulation of E2F target genes can be associated with poor prognosis in a select subset of breast cancer cases (18). The basis for these effects on breast cancer remains largely 
A

A MCF7 donor 1
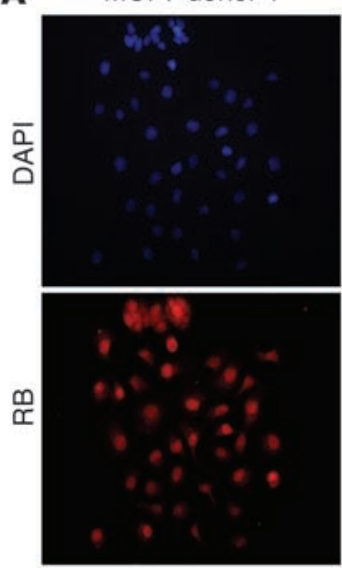

C

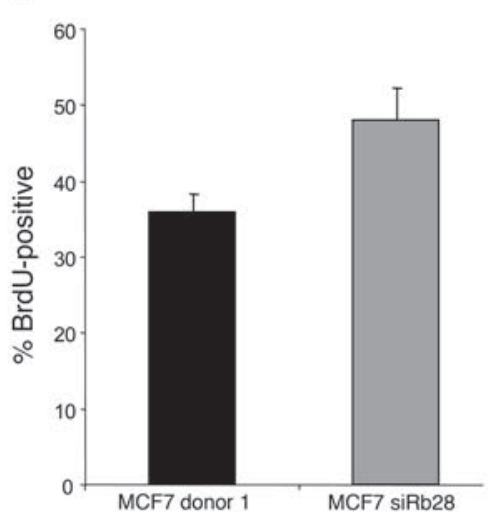

D

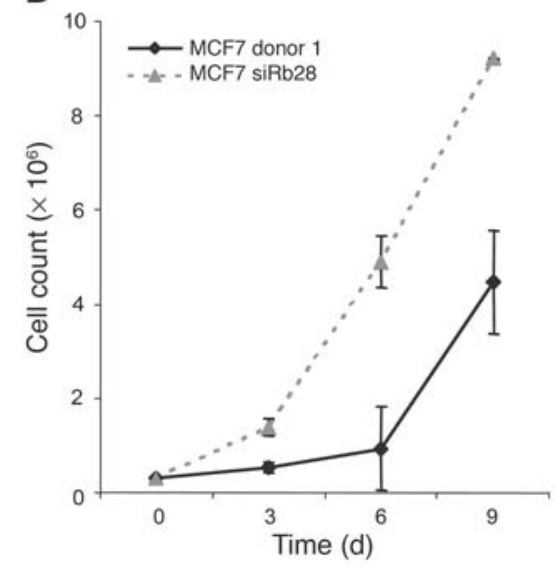

E

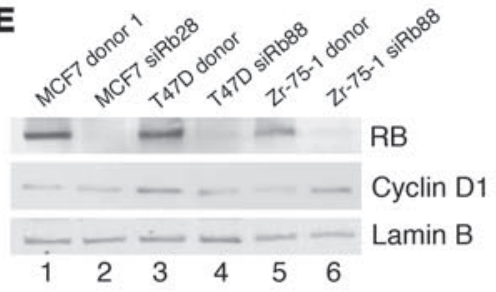

$\mathbf{F}$

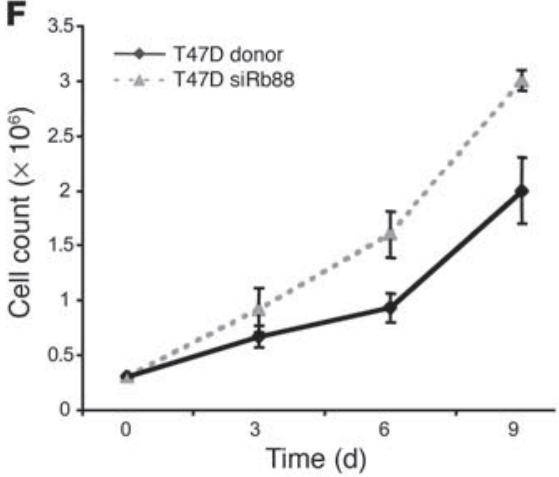

B

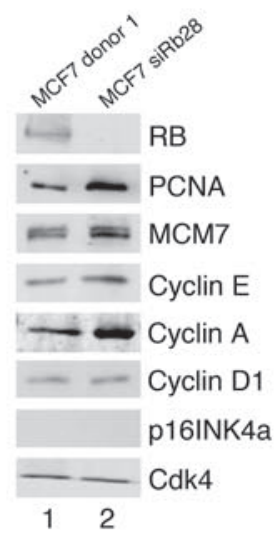

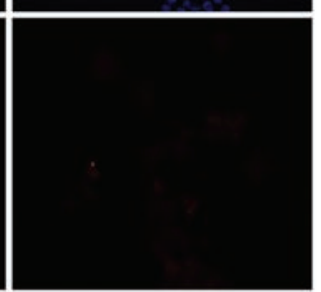

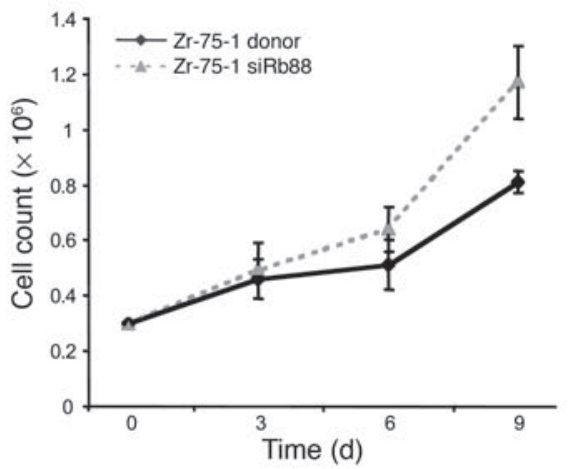

Figure 1

Efficient RB knockdown in breast cancer cells causes deregulation of RB/E2F target genes and increased proliferation kinetics. (A) MCF7 cells transfected with MSCV donor or MSCV siRb plasmids were selected with puromycin to isolate stable clones. Clones were screened by RB immunofluorescence as shown for MCF7 donor 1 and siRb28. Images were captured at equal exposures. Original magnification, $\times 20$. (B) Lysates from MCF7 donor 1 and siRb28 clones were immunoblotted for expression levels of RB, PCNA, MCM7, cyclin E, cyclin A, cyclin D1, and p16INK4a. Cdk4 served as a loading control. (C) Cells represented in $\mathbf{A}$ were BrdU labeled for 10 hours, and BrdU immunofluorescence was performed and scored. (D) Cells represented in $\mathbf{A}$ were seeded at $3 \times 10^{5}$, cell growth assays were carried out for 9 days, and cells were counted every 3 days. (E) Lysates represented in $\mathbf{B}$ along with lysates from polyclonal populations of T47D and Zr-75-1 cells infected with retrovirus encoding donor or siRb88 plasmids were immunoblotted for expression levels of RB and cyclin D1. Lamin B served as a loading control. (F) Retrovirally infected T47D and Zr-75-1 cells represented in $\mathbf{E}$ were seeded at $3 \times 10^{5}$, and growth assays were carried out as described for $\mathbf{D}$.

ruption of RB function in breast cancer, an understanding of the effect of this event upon the response to therapeutic agents is necessary for the optimal design of treatment strategies. Here, we show that RB knockdown in breast cancer cells resulted in deregulation of E2F-regulated genes and a growth advantage in vitro, which was recapitulated by accelerated tumor development in xenograft models. The effect of RB knockdown was determined in the context of radiation, cisplatin (cis-diamminedichloroplatinum II [CDDP]), or hormone ablation therapy. RB deficiency enabled cells to inappropriately progress through the cell cycle following challenge with all therapeutic modalities tested. In the context of DNA-damaging therapeutics, RB deficiency increased therapeutic sensitivity in both cell culture and xenograft models. In contrast, following Tam therapy, the bypass of cell-cycle inhibition enabled proliferation in the presence of therapy and corresponding therapeutic unknown and has not been explicitly investigated with respect to breast cancer therapies. In this regard, there is clear evidence for context-dependent effects of RB, which could have implications for the therapeutic strategies employed.

Resistance to conventional therapy is one of the main causes of patient death associated with breast cancer. Given the frequent dis- failure in xenograft models. Like RB deficiency, ectopic expression of E2F3 bypassed the cell-cycle arrest mediated by therapeutic agents, suggesting that deregulated E2F activity underlies the changes in therapeutic response in RB-deficient breast cancer cells. Using a signature of 59 RB-regulated genes to probe microarray data from breast cancer patients treated with Tam, we revealed 

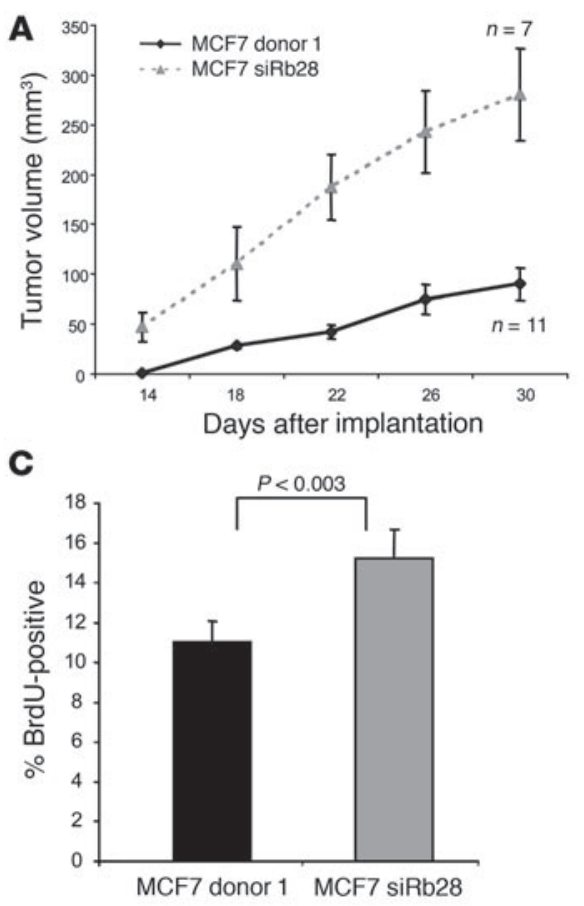

B 30 days after implantation
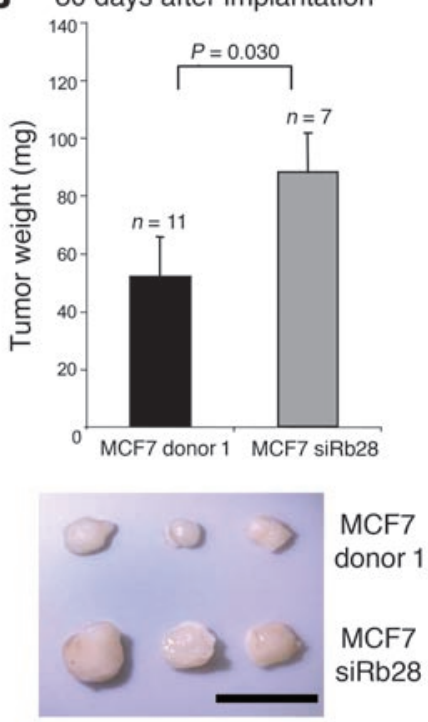

\section{Figure 2}

Tumor growth in nude mouse xenografts is accelerated in RB-knockdown cells. (A) MCF7 donor 1 or siRb28 cells were harvested and resuspended in 3:1 PBS/ Matrigel mixture. Cells $\left(2 \times 10^{6}\right)$ in $150 \mu$ of mixture were injected subcutaneously in a contralateral manner in flanks of ovariectomized nude mice. Mice were implanted with E2 pellets, and tumors were measured every 4 days. (B) Excised tumors were weighed 30 days after implantation. Tumor weights were plotted, and a 2-tailed Student's $t$ test assuming unequal variances was used to determine significance. Below: relative tumor size after excision 30 days following implantation. Scale bar: $1 \mathrm{~cm}$. (C) Nude mice represented in A were injected with BrdU 1 hour prior to sacrifice. Sectioned tumors were immunohistochemically stained and scored for BrdU incorporation, and statistical analyses were carried out as described for $\mathbf{B}$. that highly elevated gene expression levels correlated with failure of Tam therapy. Together, these results demonstrate that aberrations in RB facilitate accelerated tumorigenic proliferation of breast cancer cells and differential response to 2 major breast cancer treatment modalities.

\section{Results}

Efficient RB knockdown confers increased growth kinetics. To investigate the influence of RB function in breast cancer, we targeted its expression in the ER-positive and estrogen-dependent breast cancer cell line MCF7. Specific and stable knockdown of RB was achieved using a vector encoding short hairpin RNA (shRNA) directed against $R b$. Multiple independent clones transfected with either the shRNA against $R b$ or vector control were isolated (Supplemental Figure 1; supplemental material available online with this article; doi:10.1172/JCI28803DS1). RB protein levels were determined using immunofluorescence and immunoblotting for RB (Figure 1, A and B, top panel). These results demonstrated that $\mathrm{RB}$ protein levels had been reduced to virtually undetectable levels, and hence the cells were considered RB deficient. Since it has been postulated that nearly all cancer cells harbor compromised RB function, we initially determined the consequence of $\mathrm{RB}$ deficiency on E2F target gene expression. Thus, levels of the well-documented RB/E2F targets proliferating cell nuclear antigen (PCNA), minichromosome maintenance 7 (MCM7), cyclin E, and cyclin A were evaluated by immunoblotting. The expression levels of all of these proteins were increased in the absence of RB. However, the levels of cyclin D1 and p16INK4a (reported to be lost in MCF7 cells; ref. 19) were not affected by the reduction in $\mathrm{RB}$ protein levels (Figure 1B). Analyses of cell-cycle progression by BrdU incorporation (Figure 1C, data from multiple clones are presented in Supplemental Figure 2) or cellular proliferation (Figure 1D) demonstrated that knockdown of RB resulted in enhanced proliferation. To determine the role of RB in the growth kinetics of additional estrogen-dependent breast cancer cell lines, RB-pro- ficient and -deficient polyclonal pools of both T47D and Zr-75-1 cells were established by infection with retroviruses encoding either an shRNA directed against $R b$ or control plasmid. RB protein levels were diminished in these cell lines to a level comparable to those evident in the clonal MCF cells transfected with shRNA directed against RB (MCF7siRb28 cells), as determined by immunoblotting. As in MCF7 cells, cyclin D1 levels were unaffected by $\mathrm{RB}$ deficiency in T47D and Zr-75-1 cells (Figure 1E). Importantly, $\mathrm{RB}$ knockdown also conferred increased growth kinetics in these cell lines (Figure 1F). Thus, attenuation of RB alters the proliferative kinetics of established breast cancer cells.

To investigate the biological consequence of $\mathrm{RB}$ inactivation in tumorigenesis, we utilized nude mouse xenografts. RB-proficient and -deficient MCF7 cells $\left(2 \times 10^{6}\right)$ were injected contralaterally into the flanks of nude mice implanted with estrogen pellets to support tumor growth. RB-knockdown cells produced measurable tumors earlier than the controls and continued to grow with faster kinetics (Figure 2A), so that by 30 days the RB-knockdown tumors had grown to more than double the size of the control tumors. At this time, mice were injected with BrdU and euthanized. Tumors were excised and weighed (Figure 2B), confirming their larger size. Additionally, BrdU immunohistochemistry demonstrated that the proliferative index was significantly higher in the RB-deficient tumors (Figure 2C). Thus, even in the context of an established breast cancer cell line, RB plays a pivotal role in modulating tumorigenic proliferation.

Abrogation of the DNA damage checkpoint in RB-knockdown cells causes increased sensitivity. The RB and $\mathrm{p} 53$ tumor suppressors are known to be critical for induction of DNA damage checkpoints, and therapies inducing DNA damage are used as an additional line of treatment for ER-positive breast cancers resistant to hormonal therapy. Therefore, we investigated the role of these tumor suppressors in the response to therapeutic doses of DNA-damaging agents. Wildtype MCF7, T47D, and Zr-75-1 cells were treated with 0,8 , or $16 \mu \mathrm{M}$ CDDP and labeled with BrdU to determine the effect of the agent 

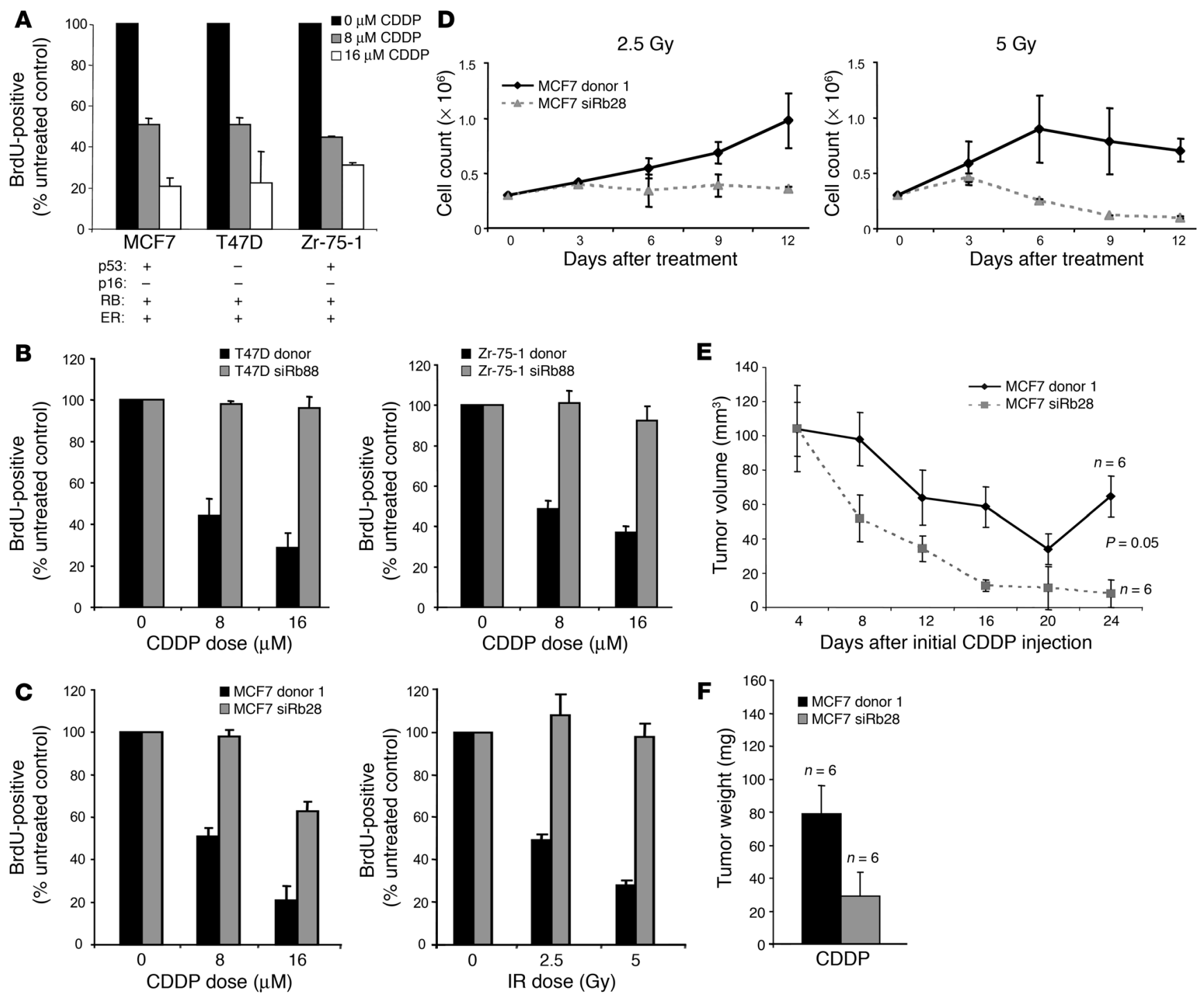

Figure 3

RB deficiency enables bypass of the DNA damage checkpoint, resulting in increased sensitivity. (A) Wild-type MCF7, T47D, and Zr-75-1 cells were treated with 0,8 , or $16 \mu \mathrm{M}$ CDDP for 18 hours, washed, and labeled with BrdU for 10 hours in culture. Cells were then fixed, and BrdU immunofluorescence was performed and scored. (B) Retrovirally infected T47D (left) and Zr-75-1 (right) donor and siRb88 cells were treated with 0 , 8 , or $16 \mu \mathrm{M}$ CDDP and BrdU labeled as described for A. (C) MCF7 donor 1 and siRb28 clones were treated with 0,8 , or $16 \mu \mathrm{M}$ CDDP for 18 hours prior to washing (left) or with $0,2.5$, or 5 Gy IR (right) and BrdU labeled as described above. (D) MCF7 donor 1 or siRb28 cells were seeded at $3 \times 10^{5}$ and treated with 2.5 (left) or 5 Gy IR (right), and cell growth assays were performed for 12 days and cells counted every 3 days. (E) Harvested MCF7 donor 1 and siRb28 cells were resuspended 3:1 in PBS/Matrigel and injected subcutaneously into the flanks of mice implanted with E2 pellets. When xenograft tumors reached approximately $110 \mathrm{~mm}^{3}$ during tumor development, mice were treated with CDDP (the E2 pellet was retained, and $5 \mathrm{mg} / \mathrm{kg}$ CDDP was injected i.p. every 4 days for 5 courses), and tumor size was monitored by caliper measurement. Tumor measurements are plotted, and a 2-tailed Student's $t$ test assuming unequal variances was used to determine significance of curves. (F) Tumors represented in $\mathbf{E}$ were weighed upon excision.

on cell-cycle progression. Although these RB-positive cell types differ in p53 status, all cell lines exhibited a dose-dependent cell-cycle checkpoint in response to CDDP treatment (Figure 3A). To specifically probe the impact of $\mathrm{RB}$ in the response to DNA damage, T47D and Zr-75-1 polyclonal siRb88 cells were utilized, with donor cells as controls. These studies demonstrated that RB-proficient T47D and Zr-75-1 cells initiated a dose-dependent checkpoint after CDDP treatment, whereas RB-deficient cells continued to incorporate BrdU efficiently following DNA damage (Figure 3B). To con- firm these findings, MCF7 donor 1 and siRb28 cells were treated either with CDDP as described above or with $0,2.5$, or 5 Gy ionizing radiation (IR). Similarly, these studies revealed that RB deficiency promoted bypass of the DNA damage checkpoints induced by both CDDP and IR treatment in MCF7 cells (Figure 3C). To determine the long-term effect of DNA damage therapy upon proliferation, cell growth assays were performed wherein the RB-proficient and -deficient MCF7 clones were plated at equal density and treated with 2.5 or 5 Gy IR. Cell counting over 12 days revealed that the 
RB-knockdown cells were more sensitive to IR following treatment with 2.5 and 5 Gy Ir relative to the donor control cells (Figure 3D). These data demonstrate that the ability of the RB-knockdown cells to progress through the cell cycle in the presence of DNA damage was associated with increased sensitivity to these agents.

Further studies of p53 function were carried out in the MCF7 RB-proficient and -deficient clones infected with either empty retroviral vector, LXSN, or LXSN-p53dd, an N-terminally truncated, dominant-negative form of murine p53. Consistent with previously published results, immunoblots revealed an accumulation of p53 evident in LXSN-p53dd-infected cells as compared with controls; however, these elevated levels were not associated with an increase in p21 following exposure to $16 \mu \mathrm{M}$ CDDP for 18 hours, suggesting that p53 function had been effectively compromised $(20,21)$ (Supplemental Figure 4A). These studies revealed that p53 dysfunction does not impact normal cell proliferation, but it does compromise the DNA damage checkpoint similarly to RB deficiency (Supplemental Figure 4, B and C). Additionally, p53 function did not significantly affect the long-term sensitivity to DNA damage imparted by IR, suggesting that RB is a more critical determinant of the therapeutic response to these types of therapies in this context (Supplemental Figure 4D).

The xenograft model system was then utilized to test the response of RB-deficient tumors to CDDP therapy. Tumors were developed in the flanks of mice by injecting MCF7 donor or siRb cells into the flanks of mice and implanting a $17 \beta$-estradiol (E2) pellet into the back. When tumors reached $100-110 \mathrm{~mm}^{3}$, mice received $5 \mathrm{mg} / \mathrm{kg}$ CDDP every 4 days for 5 courses. These experiments revealed that both tumor types regressed during CDDP treatment; however, the RB-deficient tumors regressed more rapidly throughout the 5 courses of therapy (Figure 3E) and failed to demonstrate any recovery following the completion of therapy. Upon excision, all tumors were weighed, and the results demonstrate that tumors lacking RB function weighed less than half as much as control tumors following CDDP therapy (Figure 3F), indicating that RB-deficient tumors respond more favorably to DNA damage therapy.

$R B$-deficient cells are able to bypass estrogen ablation therapy. To determine the effect of first-line breast cancer antiestrogen therapies on $\mathrm{RB}$ function, cells were exposed to several therapeutically relevant conditions. Specifically, wild-type MCF7, T47D, and Zr-75-1 cells were cultured in medium containing either FBS or charcoal dextran-treated (CDT) serum in addition to Tam, so as to antagonize ER function. In all cell lines, cyclin D1 levels decreased following 72 hours of hormone deprivation, yielding RB dephosphorylation despite undetectable levels of p16INK4a. U2OS and SaOS2 cells were included as controls for RB and p16INK4a expression levels, respectively (Figure 4A). To understand the impact of RB deficiency on the response to hormone deprivation therapy, cells were cultured in CDT; CDT plus Tam; or CDT plus the antiestrogen ICI 182780 (ICI). Following treatment with these modalities, cells were labeled with BrdU to determine the influence of each agent on cell-cycle progression and the corresponding influence of RB knockdown on this response (Figure 4B; data from multiple clones are presented in Supplemental Figure 3). These experiments were recapitulated in the polyclonal populations of T47D and $\mathrm{Zr}-75-1$ donor and siRb88 cells (Figure 4C). All conditions limiting estrogen function elicited cell-cycle inhibition in cells harboring functional RB. However, this action of each agent was significantly reduced by the depletion of RB. These results indicate that RB-deficient cells are able to partially bypass the cell-cycle blockade elicited by antiestrogen therapy. To elucidate the long-term growth effects of these therapies, cell proliferation assays were performed over 9 days. RB-proficient and -deficient MCF7 cells were seeded at equal densities and cultured in CDT/Tam (Figure 4D). As previously described, control MCF7 cells did not exhibit cell proliferation (2). However, cells lacking RB were able to continue to proliferate in this hormone-deprived environment. Similar results were evident in the same experiments with both T47D and Zr-75-1 RB-proficient and -deficient polyclonal pools (Figure 4E). To assess the role of $\mathrm{RB}$ in the therapeutic response of MCF7 cells in vivo, the xenograft model was again employed. Upon the attainment of tumors of approximately $100-120 \mathrm{~mm}^{3}$, mice were deprived of estrogen and treated with Tam. Tumor measurements taken at 4-day intervals demonstrated that RB-proficient tumors respond to Tam by regressing to nearly immeasurable sizes (Figure 4F). However, the RB-deficient counterparts did not regress and, indeed, increased in size from approximately $110 \mathrm{~mm}^{3}$ to $150 \mathrm{~mm}^{3}$ in the presence of Tam. All tumors were weighed upon excision, and RB-deficient tumors were more than 3-fold heavier than control tumors following Tam therapy (Figure 4G). Together, these data demonstrate that hormone deprivation therapy is compromised in breast cancers harboring functional inactivation of the RB pathway.

$R B$ target gene upregulation is a prognostic indicator in buman breast cancers. RB performs a myriad of functions, the most well understood being repression of the E2F family of transcription factors. To determine the specific influence of the E2F axis on bypassing therapy, an activator E2F was overexpressed in wild-type MCF7 cells. Specifically, cells were infected either with an adenovirus encoding E2F3 (Ad-E2F3) or a control virus (Ad-LacZ) and were harvested 3 days after infection for immunoblot analysis of levels of known RB-E2F targets (Figure 5A). Relative to control (lane 1), the Ad-E2F3-infected MCF7 cells (lane 2) exhibited significantly increased protein levels of E2F target genes, including PCNA and MCM7. As expected, no changes were detected in RB levels, and CDK4 served as a loading control. In order to assess the response to therapeutic intervention, 3 days after infection Ad-E2F3- or Ad-LacZ-infected MCF7 cells were separated into 2 major treatment groups: hormone therapy and DNA damage therapy. The estrogen ablation group was then cultured in the absence of estrogen and in the presence of Tam or ICI as described above. Alternatively, the cells in the DNA damage therapy group were treated with $16 \mu \mathrm{M}$ CDDP for 18 hours prior to washing or treatment with 5 Gy IR. Cells from both therapy groups were BrdU labeled, and the replicative fraction of treated cells was determined with respect to untreated control cells (Figure 5B). Cells overexpressing E2F3 exhibited significantly reduced levels of cell-cycle arrest in each therapeutic condition as compared with the control infected cells. This result indicates that the ability of RB-deficient breast cancer cells to bypass therapeutic cell-cycle arrest is due to unrestrained E2F activity, suggesting that RB/E2F-regulated target gene expression is an important marker of therapeutic response.

To determine the significance of RB/E2F target gene expression in human breast cancer, we analyzed a tumor microarray data set representing 60 breast cancer patients with ER-positive disease who were treated with Tam monotherapy. The tumor specimens in all cases had been both micro- and macrodissected (22). A gene expression signature of 59 genes that are deregulated with RB genetic loss and repressed upon RB activation was utilized to cluster this 120-point data set (60 tumors, macro- and microdissected) and is displayed as a condition tree (Figure 6A). This map shows 

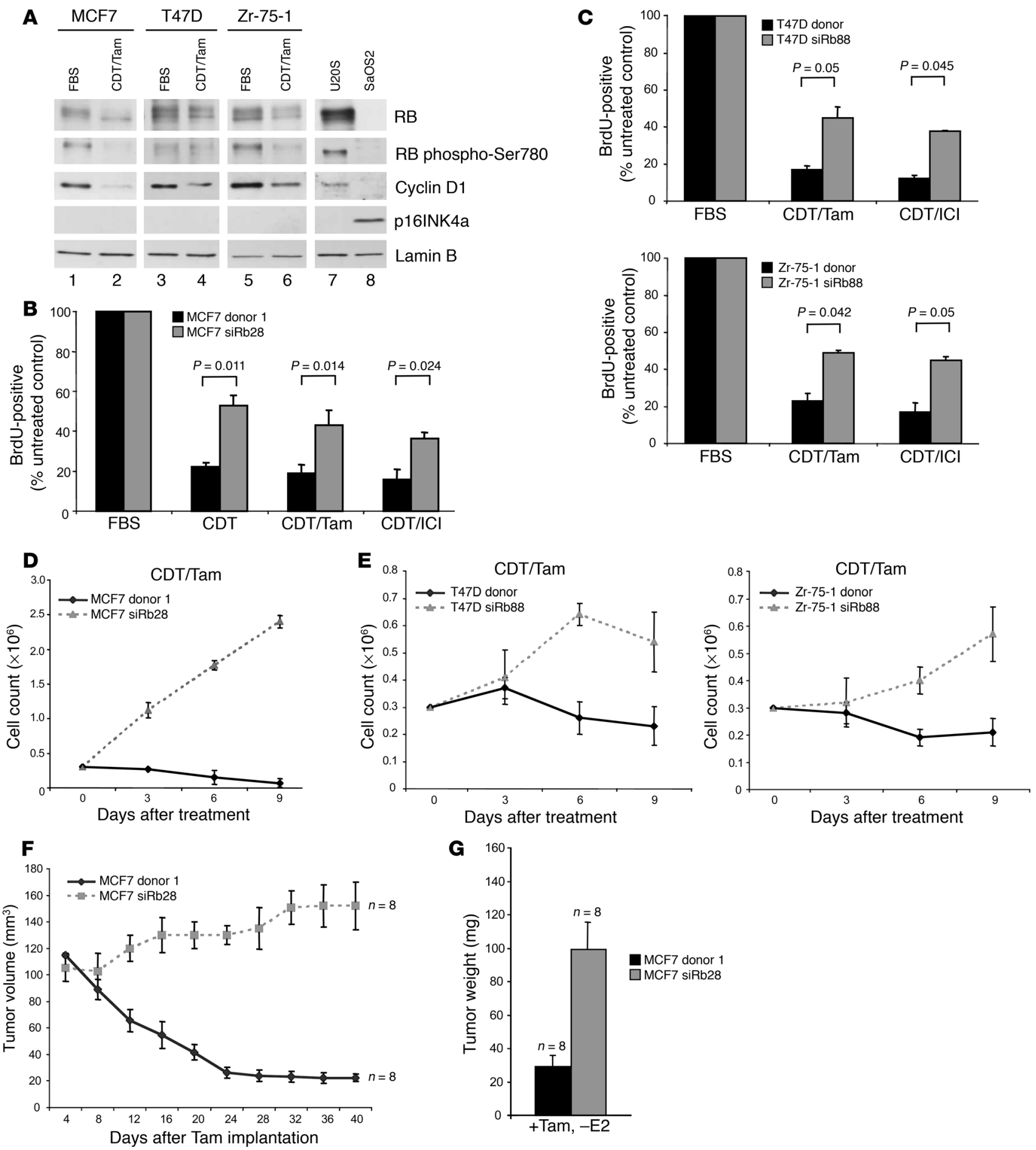

Figure 4

$\mathrm{RB}$ is necessary for sensitivity to antiestrogen therapy and long-term growth arrest. (A) Lysates from wild-type MCF7, T47D, and Zr-75-1 cells were immunoblotted for the expression levels of RB, RB phospho-Ser780, cyclin D1, and p16INK4a. Lamin B served as a loading control, while lysates from U2OS and SaOS2 cells were included as controls for RB and p16INK4a expression, respectively. (B) MCF7 donor 1 and siRb28 clones were cultured in media containing FBS, CDT, CDT/Tam, or CDT/ICl for 3 days and were BrdU labeled for the final 10 hours of culture. Cells were then fixed, and BrdU immunofluorescence was performed and scored. A 2-tailed Student's $t$ test assuming unequal variances was utilized to determine significance. (C) T47D (left) and Zr-75-1 (right) donor and siRb88 cells were cultured, BrdU labeled, and scored as described for B. Statistical tests were performed as described for B. (D) MCF7 donor 1 or siRb28 cells were seeded at $3 \times 10^{5}$, and cell growth assays were performed for 9 days while cells were cultured in CDT/Tam and counted every 3 days. (E) T47D (left) and Zr-75-1 (right) donor and siRb88 cells were seeded at $3 \times 10^{5}$, and cell growth assays were performed as described for $\mathbf{D}$. (F) When xenograft tumors (as in Figure $3 \mathrm{E}$ ) reached 100-120 mm³ , mice were treated with Tam (E2 pellet was removed and Tam pellet was added). Tumor size of the Tam-treated animals was monitored by calipers. (G) Final tumor weights of all tumors represented in $\mathbf{F}$ upon excision. 
3 major regions of gene coregulation: low, medium, and high RB target gene expression (blue, yellow, and red regions, respectively). This clustering placed the macro- and microdissected samples from 59 of 60 patients in the same gene expression groups, with only 1 tumor signature split between the high and medium gene expression groups on the condition tree.

First, correlations of the RB gene signature with known effectors of breast cancer growth or development were assessed for relevance to the RB signature. Only 3 tumors within the cohort exhibited HER2/neu positivity (labeled as a, b, and c in Figure 6A), thus indicating that HER2 status does not underlie the observed changes in gene expression pattern. Relative expression of $R b$ and $p 16 i n k 4 a$ was also examined, and mRNA levels showed no significant alteration across the tumor specimens. This result is not unexpected, as relative $R b$ and p16ink $4 a$ mRNA levels are typically unaltered even in tumors with loss of heterozygosity at the RB locus and/or that score histologically negative for RB expression (e.g., in small cell lung carcinoma; data not shown) (23). These observations are consistent with the established observations that RB inactivation is frequently associated with regulation of protein function rather than loss of the RB gene itself in breast cancer. Since one suggested mechanism for loss of RB function is attributed to excessive $G_{1}$ cyclin/CDK activity, relative $G_{1}$ cyclin mRNA levels were analyzed in the cohort. As shown, cyclin D1 levels did show a differential expression pattern, wherein a large number of tumors (43\%) showed elevations in cyclin D1 mRNA production. However, cyclin D1 status did not correlate with the RB gene expression signature, thus indicating that the major mechanism of RB inactivation in this tumor cohort is unlikely to result from enhanced cyclin D1 gene expression. Moreover, these data indicate that the RB signature is not a general consequence of cell-cycle aberrations. Cyclin $E$ mRNA levels did show an inverse relationship with functional RB, consistent with existing literature (24), and correlated with the high RB gene expression group. Together, these data indicate that the RB-deficient signature is specific and likely arises from functional inactivation of the tumor suppressor action.

To address the importance of the RB deficiency signature on therapeutic response, patient outcome was examined. Recurrence data revealed that the patients in the "high" target gene group have an increased incidence of cancer recurrence $(65 \%)$ relative to the patients in the other 2 groups (38\%). The increased levels of RB/E2F target genes in this high group would suggest that these tumors are functionally disrupted for the RB pathway, and their poor response to Tam therapy would correspond with data described herein. The average expression levels of these $59 \mathrm{RB}$ target genes in each of the 3 groups are quantitatively displayed as a box plot to reveal an approximate 3 -fold increase in gene expression from the low to the high group (Figure $6 \mathrm{~B})$. For each patient, the time to disease recurrence is known, and a recurrence-free survival curve was generated (Figure 6C). Patients responded poorly to Tam in the high expression group, wherein the median recurrence-free survival was 62.5 months, and only $35 \%$ remained recurrence free. In contrast, patients in the low/medium RB target gene expression groups had an improved prognosis on Tam therapy, with greater than $62 \%$ remaining disease

Figure 5 free. Thus, compromised control of the RB/E2F axis is associated with poor response to Tam in human breast cancer.

\section{Discussion}

The RB tumor suppressor is functionally inactivated in a large fraction of human cancers (25). Traditionally, this event is associated with the genesis of cancer as opposed to the effect on the therapeutic response of a given tumor type. Here we evaluated the influence of RB deficiency in the context of established breast cancer cells and found that while attenuation of RB function did accelerate cellular and tumorigenic proliferation, it also had a profound influence on the response to key therapeutic modalities utilized in the treatment of breast cancer.

It is widely held that $\mathrm{RB}$ functions as a negative regulator of cell-cycle progression that is targeted at high frequency in human cancers by a myriad of mechanisms. The frequency of this event has led to the hypothesis that most cancers functionally inactivate RB during tumor progression (25-27). Such a model would suggest that although the RB protein is expressed in many tumor types, it is functionally inert due to upstream deregulation of $\mathrm{RB}$ phosphorylation. In this work, we investigated the influence of RB deficiency on several breast cancer cell lines. We found that knockdown of RB led to the enhanced expression of E2F target genes, suggesting that the RB protein present in MCF7 cells is at least partially functional. Subsequent analyses of cell-cycle progression and cellular proliferation indicated that, similar to what is observed in primary cells, knockdown of RB induces a modest proliferative advantage in MCF7, T47D, and Zr-75-1 cells (28). Strikingly, in MCF7 cells, RB deficiency also facilitated tumorigenic proliferation. Thus, while many mechanisms may attenuate the RB pathway sufficiently to promote tumorigenesis, many tumor lines retain sufficient RB activity to activate checkpoints when provoked, such that complete deficiency of RB can further enhance proliferation and alter tumor cell behavior.
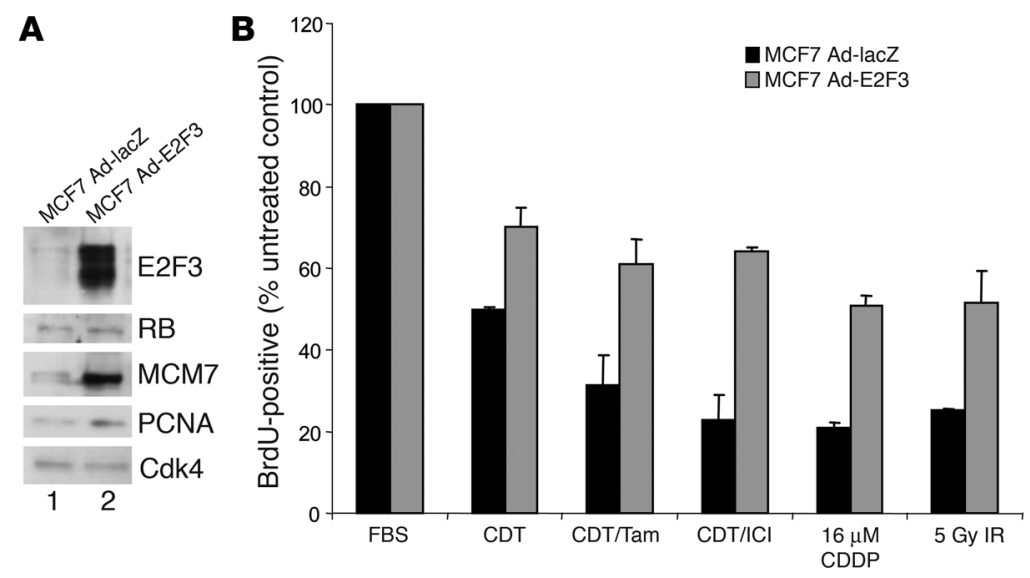

E2F3 overexpression in MCF7 cells allows bypass of antimitogenic checkpoints. (A) MCF7 cells infected with adenoviral vectors encoding either lacZ or E2F3 were harvested 3 days after infection, lysed, separated by SDS-PAGE, and immunoblotted for determination of E2F3, RB, MCM7, and PCNA expression levels. Cdk4 served as a loading control. (B) The adenovirus-infected cells represented in A were cultured in media containing FBS, CDT, CDT/Tam, or CDT/ICl for 3 days or were treated as described above with $16 \mu \mathrm{M}$ CDDP or 5 Gy IR prior to BrdU labeling for 10 hours. Cells were then fixed, and BrdU immunofluorescence and scoring were performed. 
A

60 breast tumors microdissected and macrodissected (120 total)

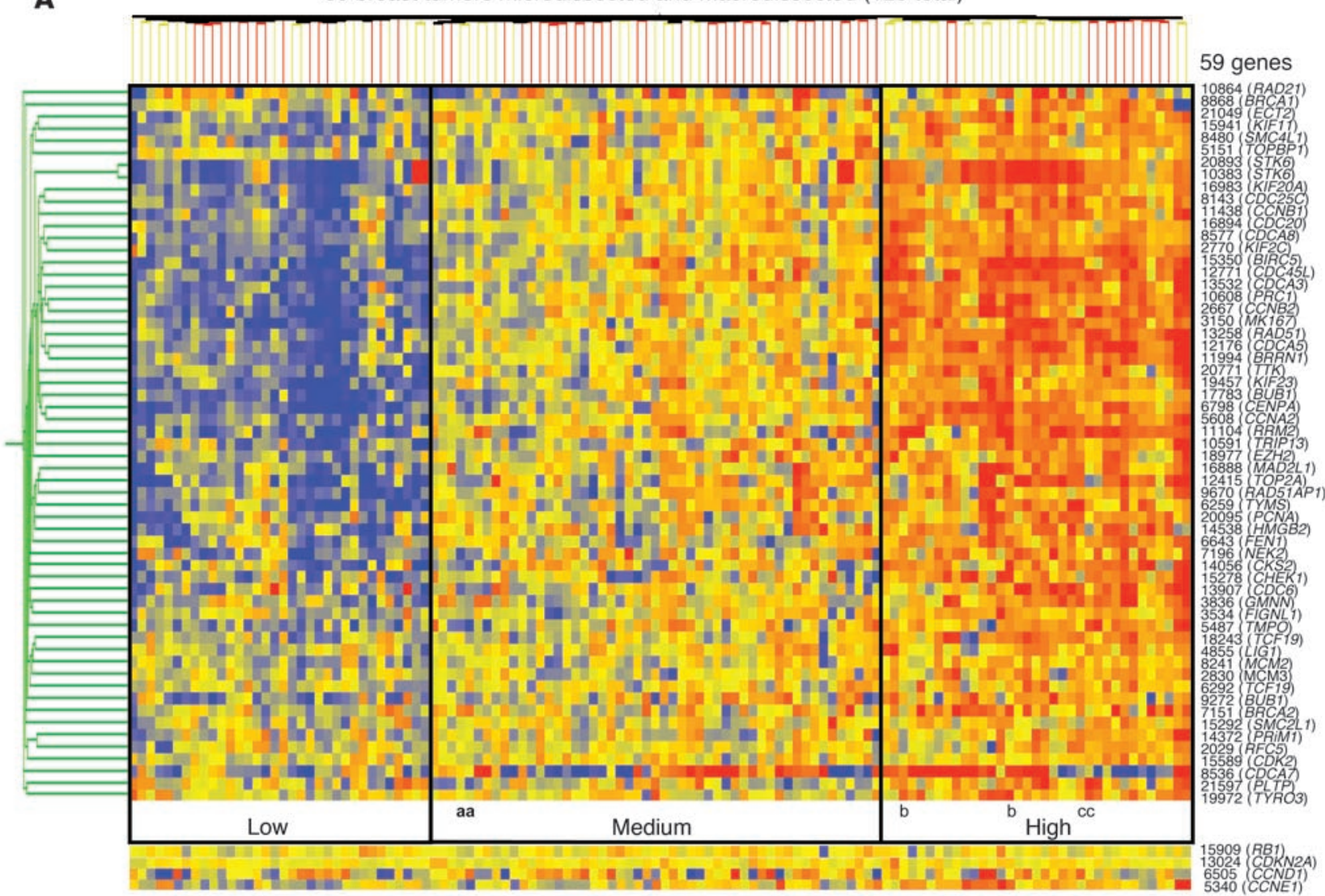

$\mathbf{B}$

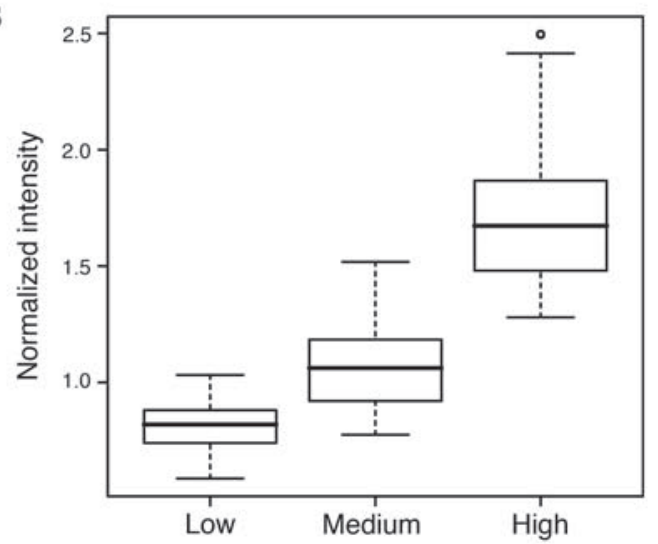

C

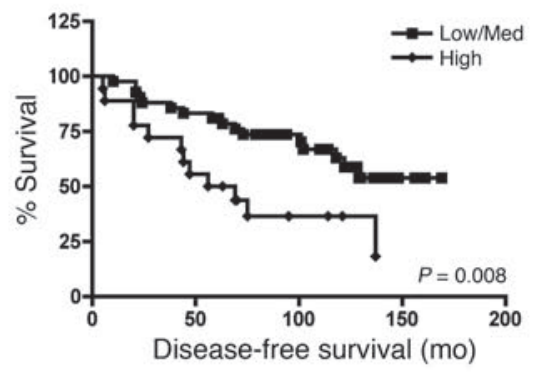

Figure 6

$\mathrm{RB} / \mathrm{E} 2 \mathrm{~F}$ downstream target deregulation correlates with poor prognosis in human breast cancers treated with Tam monotherapy. (A) Gene expression data from 60 ER-positive human breast tumors that were both micro- and macrodissected were analyzed for RB/E2F target gene expression using GeneSpring. The expression patterns of 59 known RB/E2F target genes and the expression levels of $R b, p 16, c y c l i n ~ D 1$, and cyclin $E$ are displayed in a condition tree for each of the 2 tissue samples from each patient (120 samples). The average RB/E2F target gene expression levels of all 59 genes were categorized into 3 groups: low, medium, and high. Three HER2/neu-positive tumors (a, b, and c) were present in this tumor set. (B) The RB/E2F target gene expression levels in each group represented in $\mathbf{C}$ were averaged and displayed as a boxand-whisker plot. A 2-tailed Student's $t$ test assuming unequal variances was utilized to determine significance $\left(P=7.2 \times 10^{-12}\right.$ low/medium; $P=1.3 \times 10^{-14}$ medium/high). (C) The survival data for each of the 60 patients from the low/medium and high gene expression groups represented in $\mathbf{C}$ was compiled into a disease-free survival curve. Statistical tests were performed as described for $\mathbf{B}$.

Breast cancer, like all cancers, is a heterogeneous disease. In the case of ER-positive disease, Tam or similar antiestrogenic compounds are utilized to treat the cancer. However, many ER-positive patients develop resistance to antiestrogen therapy, and an alternative line of therapy such as either radiation or chemotherapy is required. It has been found in primary cells that the RB/E2F pathway plays an important role in mediating cell-cycle inhibition following exposure to such agents $(29,30)$. In several breast cancer cell lines, we 
observed a similar dependence on RB/E2F function, as either the knockdown of RB or overexpression of E2F3 (MCF7 cells) enabled bypass of the DNA damage checkpoint. Strikingly, this loss of checkpoint function increased the sensitivity of RB-deficient MCF7 cells or tumors to IR and CDDP, respectively. These findings are in agreement with prior studies in primary murine cells, where RB deficiency was found to enhance susceptibility to death following DNA damage $(31,32)$, which was most likely due to deregulation of cell-cycle and proapoptotic genes $(33,34)$. Thus, aberrations in $\mathrm{RB}$, while uncoupling cell-cycle responses, lead to enhanced sensitivity to cytotoxic therapeutics that function by damaging DNA. This is clinically important for patients harboring either ER-negative or ER-positive Tam-resistant tumors. In addition, disruptions in the p53 pathway are known to occur in $20 \%-40 \%$ of breast cancers, although most frequently in ER-negative cases (35). Therefore, the influence of p53 function on DNA damage checkpoint response and sensitivity in MCF7 models was examined, and these analyses showed that while p53 does impact the DNA damage checkpoint, there was only a marginal effect on long-term sensitivity, as suggested in the literature (36). These data indicate that RB function modifies therapeutic response even in the absence of p53.

First-line therapy for ER-positive breast cancer exploits the estrogen dependence of these cells. Treatment of estrogen-sensitive ERpositive tumors with estrogen antagonists results in inhibition of tumor growth (37) and corresponding tumor regression. However, up to $50 \%$ of ER-positive tumors fail to respond to such therapeutics (38). Here we determined the influence of compromising the $\mathrm{RB} / \mathrm{E} 2 \mathrm{~F}$ pathway on response to estrogen antagonists. Cells with a disruption in this pathway failed to undergo cell-cycle inhibition following hormone therapy. However, unlike the situation with DNA-damaging agents, the RB-deficient breast cancer cells continued to proliferate in the presence of Tam. As a result, RB-deficient tumors continued to progress in the presence of Tam and thus failed therapy. However, RB deficiency only partially bypassed the requirement for estrogen. This finding is likely attributed to the complexity of estrogen signaling pathways, which cannot be fully recapitulated by $\mathrm{RB}$ deficiency $(39,40)$. Such a supposition is supported by partial bypass that is observed with overexpression of HER2/neu (41). Interestingly, although p53-deficient MCF7 cells were able to bypass the DNA damage checkpoint, the same was not evident following hormone ablation therapy (Supplemental Figure 4E). These data, combined with the observation that MCF7, T47D, and Zr-75-1 cells are all p16INK4a deficient (19) yet display resistance to hormone therapy upon RB knockdown, suggest that the influence of RB on the response to antiestrogen therapy is not effectively recapitulated by other tumor suppressor pathways. Together, these findings have significant clinical impact, since the majority of tumors that initially respond to Tam eventually develop cellular resistance (42).

The involvement of RB function in breast cancer therapy has not previously been examined. In human disease, disruption of the RB pathway occurs with relatively high frequency $(>80 \%)(13,17)$ and is often associated with poor prognosis. Since RB function can be disrupted via mechanisms that do not directly target the protein (e.g., point mutations) and deregulated E2F activity could similarly bypass Tam, we reasoned that analyses of RB target genes could result in an important determinant of Tam response. These RB target genes have been defined based on RB genetic loss and induction of RB function in cells. As expected, this "signature" is largely consistent with genes that are also regulated by the E2F family of transcription factors. Supporting such analyses, high levels of the $\mathrm{RB} / \mathrm{E} 2 \mathrm{~F}$ targets cyclin A and cyclin $\mathrm{E}$ have been reported to correlate with Tam resistance $(43,44)$. Furthermore, we found that high expression of RB target genes was associated with poor response to Tam in the context of monotherapy. Therefore, these clinical data indicate that disruption of the RB/E2F pathway plays a role in the progression of breast tumors to antiestrogen resistance. It could be reasoned that upregulation of $\mathrm{RB} / \mathrm{E} 2 \mathrm{~F}$ targets, many of which are cell-cycle genes, could be associated with poor prognosis in general. However, in studies to define gene expression signatures in breast cancer survival, metastasis, and tumor grade, RB/E2F target genes have not been widely represented (45-48), except in highly specific subpopulations of patients (18). Interestingly, RB targets from our signature do consistently constitute a small subset of these gene lists and thus should be subject to further investigation, as they could contribute not only to therapeutic bypass but disease severity and aggressiveness. In fact, aberrations in RB have been documented to correspond with ER negativity in breast cancers $(16,49,50)$, indicating that RB functional inactivation could be a crucial step in the progression to advanced disease. There are a myriad of mechanisms through which RB functional inactivation could occur to deregulate gene expression in breast cancer. Our studies suggest that cyclin D1 gene upregulation or protein overexpression (data not shown) fail to predict Tam response. This is consistent with several studies that have shown that cyclin D1 levels do not stringently correlate with Tam resistance $(51,52)$. Although our studies are modeling immunohistochemical loss of RB, disruption of RB transcriptional repression can occur through additional mechanisms that could also contribute to Tam resistance. For example, excessive cyclin E expression, expression of hyperactive (low-molecular-weight) cyclin E, loss of the CDK2 inhibitor p27kip1, or loss of the Brm SWI/SNF ATPase have been shown to compromise RB function in cancer cells $(44,53,54)$. The proximal influence of RB on target gene deregulation remains under investigation, and an understanding will be critical for refining application of the RB signature. Together, these studies strongly suggest that disruption of the RB/E2F axis has a deleterious influence on hormone therapy and could be utilized as a metric for informing therapeutic choice.

\section{Methods}

Cell lines, viral infections, and culture. The MCF7, T47D, and Zr-75-1 cell lines were obtained from ATCC and propagated in DMEM containing 10\% FBS supplemented with $100 \mathrm{U} / \mathrm{ml}$ penicillin/streptomycin and $2 \mathrm{mM}$ L-glutamine at $37^{\circ} \mathrm{C}$ in air containing $5 \% \mathrm{CO}_{2}$. Cells were infected with adenovirus encoding either E2F3 (Ad-E2F3 was a generous gift from James DeGregori, University of Colorado Health Sciences Center, Aurora, Colorado, USA) or the lacZ gene (Ad-lacZ was kindly supplied by Nancy Ratner, Cincinnati Children's Hospital Medical Center) as a control. Cells were infected with Ad-lacZ at a multiplicity of infection of 50 , at which $95 \%$ of cells were infected (as determined by plaque assay in 293 cells) or with $2.7 \times 10^{11}$ virus particles/ml of Ad-E2F3 and cultured for 3 days prior to use. RB-knockdown or control MCF7 cells were created through transfection with either an shRNA plasmid directed against $R b$ (MSCV-Rb3C; targeted sequence: 5'-CGCATACTCCGGTTAGGACTGTTATGAA-3') or a control plasmid (MSCV donor) using FuGENE Transfection Reagent (Roche Applied Science). Following selection with $2.5 \mu \mathrm{g} / \mathrm{ml}$ puromycin for 3-4 days, stable clones were isolated and characterized. Retrovirus encoding an shRB plasmid (MSCV-LMP Rb88; targeted sequence: 5'-GAAAGGACATGTGAACTTA-3') or control plasmid (MSCV donor) were utilized to create RB-knockdown or control Zr-75-1 and T47D cell lines. Following selection 
with $2.5 \mu \mathrm{g} / \mathrm{ml}$ puromycin for 3-4 days, polyclonal cell lines were characterized. MCF7 donor 1 and MCF7siRb28 cells were infected with retrovirus encoding LXSN or LXSN-p53dd (generously provided by Susanne Wells, Cincinnati Children's Hospital Medical Center) (55), selected with 900 $\mu \mathrm{g} / \mathrm{ml} \mathrm{G} 418$ for 7 days, and pooled for characterization.

To study the effect of estrogen depletion, cells were cultured for 72 hours in phenol red-free DMEM supplemented with $10 \%$ CDT serum with addition of $10^{-9} \mathrm{M}$ 4-HydroxyTam (Sigma-Aldrich) or $10^{-6} \mathrm{M} \mathrm{ICI}$ (Tocris Bioscience) where indicated. Cell growth assays were performed by trypsinizing cells and counting by trypan blue exclusion every 3 days.

Immunoblot analysis, immunofluorescence, and DNA damage. Cells were harvested by trypsinization and lysed in RIPA buffer. Equal amounts of protein, as determined by Bio-Rad DC assay, were resolved by SDS-PAGE. Specific proteins were detected by standard immunoblotting procedures using the following primary antibodies: p16INK4a (F-12; 1:500 dilution; Santa Cruz Biotechnology Inc.), cyclin D1 (H295), PCNA (pc10), cyclin E (HE12), cyclin A (C-19), MCM7 (141.2), E2F3 (C-18), cdk4 (H-22), lamin B (M-20), p21 (C-19), p53 (Ab-6; 1:500 dilution; Calbiochem; EMD Biosciences), RB phospho ser 780 (9307S; 1:500 dilution; Cell Signaling Technology), and anti-RB (G3-245; 1:100 dilution; BD Biosciences). Immunofluorescence staining for RB was performed on cells growing on coverslips by fixing them in $3.7 \%$ formaldehyde in PBS for 10 minutes. Cells were permeabilized in $0.3 \%$ Triton X-100 in PBS for 20 minutes and blocked in 5\% fetal goat serum in PBS for 1 hour. Cells were incubated in blocking solution with anti-RB antibody (G3-245; 1:25 dilution; BD Biosciences) for 1 hour at $37^{\circ} \mathrm{C}$ followed by PBS washing and incubation with Alexa Fluor $488-$ conjugated secondary antibody (1:100; Invitrogen) and counterstained with DAPI (Sigma-Aldrich).

Cells treated with IR were exposed to ${ }^{137} \mathrm{Cs}$ (dose rate: $0.67 \mathrm{~Gy} / \mathrm{min}$ ) at room temperature in tissue culture media. Culture with clinical grade CDDP for 18 hours was performed for all CDDP treatments. For all proliferation studies, cells were labeled with BrdU for 10 hours or 5 hours for LXSN/LXSN-p53dd infected cells, and BrdU immunofluorescence was performed as previously described (56). All BrdU results are expressed as a percentage of untreated control cells set to $100 \%$, unless otherwise noted.

Xenograft studies and immunohistochemistry. Five- to 8-week-old female ovariectomized athymic nude mice (Harlan) were anesthetized, and an E2 pellet (1.7 mg/pellet, 90-day release; Innovative Research of America) or placebo was surgically implanted in the back. Following implantation, $200 \mu \mathrm{l}$ of a PBS-and phenol red-free Matrigel matrix basement membrane (BD Biosciences), solution (3:1) containing $2 \times 10^{6}$ cells was injected subcutaneously into the flank, or contralaterally where noted. Tumor volume was measured with calipers every 4 days using the equation $V=0.52$ (width $)^{2} \times$ (length). In the therapeutic studies, when tumor volume reached $100-120 \mathrm{~mm}^{3}$, animals were placed into 1 of 3 therapeutic groups (each group containing at least 6 animals): control animals retaining the estrogen pellet, antiestrogen-treated animals, or CDDP-treated animals. Animals in the antiestrogen treatment group were anesthetized, the estrogen pellet was surgically removed, and a Tam pellet ( $5 \mathrm{mg} /$ pellet, 60 -day release; Innovative Research of America) implanted. The CDDP-treated animals received $5 \mathrm{mg} / \mathrm{kg}$ CDDP injected i.p. on a $\mathrm{q} 4 \mathrm{dx} 5$ schedule (every 4 days for 5 courses). All animals were injected i.p. with $150 \mathrm{mg} / \mathrm{kg} \mathrm{BrdU}$ (Sigma-Aldrich) 1 hour prior to euthanization. Xenograft tumors were weighed and fixed in $10 \%$ neutral buffered formalin, paraffin embedded, and cut into 5 - $\mu \mathrm{m}$ sections. For immunohistochemical staining, sections were deparaffinized in xylene and rehydrated through a graded series of ethanol/water solutions. A BrdU detection kit (Zymed) was utilized as recommended by the manufacturer. BrdU incorporation was scored in a blinded manner, and at least 500 cells per section were counted from several random fields. All animal experimentation was conducted in accordance with the NIH Guide for Care and Use of Laboratory Animals and was approved by the University of Cincinnati Institutional Animal Care and Use Committee.

Microarray analysis. Microarray data (series GSE1378 and GSE1379) from X.J. Ma et al. (22) were obtained from the National Center for Biotechnology Information Gene Expression Omnibus (GEO) website (http://www. ncbi.nlm.nih.gov/geo/query/acc.cgi?acc $=$ GPL1223) and manipulated using GeneSpring GX software (version 7.2) (Agilent Technologies). For each series, the raw data was obtained from GEO as $\log _{2}$ of normalized $\mathrm{Cy} 5 / \mathrm{Cy} 3$ ratio, where tumor sample RNA and human universal reference RNA were labeled with Cy5 and Cy3, respectively. The raw data were transformed from $\log _{2}$ to linear values followed by per-gene median normalization in GeneSpring. A 120-sample experiment was then created by combining the normalized data of the 2 series (60 tumors, macro- and microdissected). The expression levels of $59 \mathrm{RB}$ target genes analyzed were derived from analyses of RB loss or gain of function in model systems. To identify the effect of RB loss on gene expression, Affymetrix gene chip analyses were performed using primary murine fibroblasts, wherein the $R b$ gene is chronically or acutely inactivated. The gain of function studies have been previously published (57). The expression levels of these 59 genes were analyzed in the combined experiment of 120 breast cancer specimens. Initially, the genes were clustered based on standard correlation as the similarity measurement. Subsequently, a condition tree based on distance correlation was created to order the tumor specimens.

The low, medium, and high expression groups were significantly distinct based on gene expression. Specifically, using a 2-tailed Welch's $t$ test, the high group was statistically distinct from the low and medium groups $\left(P=2.3 \times 10^{-19}\right.$ and $\left.P=1.3 \times 10^{-14}\right)$, and the medium group was statistically distinct from the low group $\left(P=7.2 \times 10^{-12}\right)$. For Kaplan-Meier analysis, statistical calculations were performed using GraphPad Prism 4.0 software, and the $P$ value presented $(P=0.008)$ was based on a log-rank Mantel-Haenzel test (Figure 6). All other results were analyzed for statistical significance using the 2 -tailed Student's $t$ test. For all analyses, $P<0.05$ was considered significant.

\section{Acknowledgments}

The authors would like to thank Chris Mayhew for thoughtful comments and critical reading of the manuscript. Pilot funding for this project was provided by generous donations to the Barrett UC Cancer Center from GE Aviation and the My Mother, My Daughter, Myself breast cancer event. E.E. Bosco is supported by the Albert J. Ryan Foundation and Department of Defense Breast Cancer Research Program Predoctoral Grant W81XWH0410329. This work was supported by NIH grants to B.J. Aronow (CA08429 and ES06096), to S.W. Lowe (AG16379), to K.E. Knudsen (CA099996), and to E.S. Knudsen (CA106471).

Received for publication April 12, 2006, and accepted in revised form October 24, 2006.

Address correspondence to: Erik Knudsen, University of Cincinnati, 3125 Eden Ave., Cincinnati, Ohio 45267, USA. Phone: (513) 558-8885; Fax: (513) 558-4454; E-mail: erik.knudsen@uc.edu.
1. Jensen, E.V., and Jordan, V.C. 2003. The estrogen receptor: a model for molecular medicine. Clin. Cancer Res. 9:1980-1989.

2. Sutherland, R.L., Green, M.D., Hall, R.E., Reddel,
R.R., and Taylor, I.W. 1983. Tamoxifen induces accumulation of MCF 7 human mammary carcinoma cells in the G0/G1 phase of the cell cycle. Eur. J. Cancer Clin. Oncol. 19:615-621.
3. Osborne, C.K., Boldt, D.H., Clark, G.M., and Trent, J.M. 1983. Effects of tamoxifen on human breast cancer cell cycle kinetics: accumulation of cells in early G1 phase. Cancer Res. 43:3583-3585. 
4. Encarnacion, C.A., et al. 1993. Measurement of steroid hormone receptors in breast cancer patients on tamoxifen. Breast Cancer Res. Treat. 26:237-246.

5. Howell, A., and Robertson, J. 1995. Response to a specific antioestrogen (ICI 182780) in tamoxifenresistant breast cancer. Lancet. 345:989-990.

6. Robertson, J.F. 1996. Oestrogen receptor: a stable phenotype in breast cancer. Br. J. Cancer. 73:5-12.

7. Broceno, C., Wilkie, S., and Mittnacht, S. 2002. RB activation defect in tumor cell lines. Proc. Natl. Acad. Sci. U. S. A. 99:14200-14205

8. Foster, J.S., Henley, D.C., Bukovsky, A., Seth, P., and Wimalasena, J. 2001. Multifaceted regulation of cell cycle progression by estrogen: regulation of Cdk inhibitors and Cdc25A independent of cyclin D1-Cdk4 function. Mol. Cell. Biol. 21:794-810.

9. Sutherland, R.L., and Musgrove, E.A. 2004. Cyclins and breast cancer. J. Mammary Gland Biol. Neoplasia. 9:95-104.

10. Carroll, J.S., Prall, O.W., Musgrove, E.A., and Sutherland, R.L. 2000. A pure estrogen antagonist inhibits cyclin E-Cdk2 activity in MCF-7 breast cancer cells and induces accumulation of p130E2F4 complexes characteristic of quiescence. J. Biol. Chem. 275:38221-38229.

11. Watts, C.K., et al. 1995. Antiestrogen inhibition of cell cycle progression in breast cancer cells in associated with inhibition of cyclin-dependent kinase activity and decreased retinoblastoma protein phosphorylation. Mol. Endocrinol. 9:1804-1813.

12. Pietilainen, T., et al. 1995. Expression of retinoblastoma gene protein $(\mathrm{Rb})$ in breast cancer as related to established prognostic factors and survival. Eur. J. Cancer. 31A:329-333.

13. Oesterreich, S., and Fuqua, S.A. 1999. Tumor suppressor genes in breast cancer. Endocr. Relat. Cancer. 6:405-419

14. Chano, T., Kontani, K., Teramoto, K., Okabe, H., and Ikegawa, S. 2002. Truncating mutations of RB1CC1 in human breast cancer. Nat. Genet. 31:285-288.

15. Borg, A., Zhang, Q.X., Alm, P., Olsson, H., and Sellberg, G. 1992. The retinoblastoma gene in breast cancer: allele loss is not correlated with loss of gene protein expression. Cancer Res. 52:2991-2994.

16. Ceccarelli, C., et al. 1998. Retinoblastoma (RB1) gene product expression in breast carcinoma. Correlation with Ki-67 growth fraction and biopathological profile. J. Clin. Pathol. 51:818-824.

17. Malumbres, M., and Barbacid, M. 2001. To cycle or not to cycle: a critical decision in cancer. Nat. Rev. Cancer. 1:222-231.

18. Dai, H., et al. 2005. A cell proliferation signature is a marker of extremely poor outcome in a sub population of breast cancer patients. Cancer Res. 65:4059-4066

19. Zhou, R., Frum, R., Deb, S., and Deb, S.P. 2005 The growth arrest function of the human oncoprotein mouse double minute- 2 is disabled by downstream mutation in cancer cells. Cancer Res. 65:1839-1848.

20. Sheard, M.A., Uldrijan, S., and Vojtesek, B. 2003 Role of p53 in regulating constitutive and X-radiation-inducible CD95 expression and function in carcinoma cells. Cancer Res. 63:7176-7184

21. Sheen, J.H., and Dickson, R.B. 2002. Overexpres- sion of c-Myc alters $\mathrm{G}(1) / \mathrm{S}$ arrest following ionizing radiation. Mol. Cell. Biol. 22:1819-1833.

22. Ma, X.J., et al. 2004. A two-gene expression ratio predicts clinical outcome in breast cancer patients treated with tamoxifen. Cancer Cell. 5:607-616.

23. Bhattacharjee, A., et al. 2001. Classification of human lung carcinomas by mRNA expression profiling reveals distinct adenocarcinoma subclasses. Proc. Natl. Acad. Sci. U. S. A. 98:13790-13795.

24. Nielsen, N.H., Emdin, S.O., Cajander, J., and Landberg, G. 1997. Deregulation of cyclin E and D1 in breast cancer is associated with inactivation of the retinoblastoma protein. Oncogene. 14:295-304

25. Sherr, C.J., and McCormick, F. 2002. The RB and p53 pathways in cancer. Cancer Cell. 2:103-112.

26. Hanahan, D., and Weinberg, R.A. 2000. The hallmarks of cancer. Cell. 100:57-70.

27. Dimova, D.K., and Dyson, N.J. 2005. The E2F transcriptional network: old acquaintances with new faces. Oncogene. 24:2810-2826.

28. Sage, J., et al. 2000. Targeted disruption of the three Rb-related genes leads to loss of $\mathrm{G}(1)$ control and immortalization. Genes Dev. 14:3037-3050.

29. Mayhew, C.N., et al. 2004. Discrete signaling pathways participate in RB-dependent responses to chemotherapeutic agents. Oncogene. 23:4107-4120.

30. Harrington, E.A., Bruce, J.L., Harlow, E., and Dyson, N. 1998. pRB plays an essential role in cell cycle arrest induced by DNA damage. Proc. Natl. Acad. Sci. U. S. A. 95:11945-11950.

31. Almasan, A., Linke, S.P., Paulson, T.G., Huang, L.C., and Wahl, G.M. 1995. Genetic instability as a consequence of inappropriate entry into and progression through S-phase. Cancer Metastasis Rev. 14:59-73.

32. Harbour, J.W., and Dean, D.C. 2000. Rb function in cell-cycle regulation and apoptosis. Nat. Cell Biol. 2:E65-E67.

33. Nahle, Z., et al. 2002. Direct coupling of the cell cycle and cell death machinery by E2F. Nat. Cell Biol. 4:859-864.

34. Bracken, A.P., Ciro, M., Cocito, A., and Helin, K. 2004. E2F target genes: unraveling the biology. Trends Biochem. Sci. 29:409-417.

35. Borresen-Dale, A.L. 2003. TP53 and breast cancer. Hum. Mutat. 21:292-300.

36. Essmann, F., Engels, I.H., Totzke, G., SchulzeOsthoff, K., and Janicke, R.U. 2004. Apoptosis resistance of MCF-7 breast carcinoma cells to ionizing radiation is independent of p53 and cell cycle control but caused by the lack of caspase- 3 and a caffeine-inhibitable event. Cancer Res. 64:7065-7072.

37. Leung, B.S., and Potter, A.H. 1987. Mode of estrogen action on cell proliferative kinetics in CAMA-1 cells. I. Effect of serum and estrogen. Cancer Invest. 5:187-194.

38. Jordan, V.C., and Morrow, M. 2003. Re: trends in use of adjuvant multi-agent chemotherapy and tamoxifen for breast cancer in the United States: 1975-1999. J. Natl. Cancer Inst. 95:683-684; author reply 684-685.

39. Osborne, C.K., Schiff, R., Fuqua, S.A., and Shou, J. 2001. Estrogen receptor: current understanding of its activation and modulation. Clin. Cancer Res. 7:4338s-4342s; discussion 4411s-4412s.

40. Nicholson, R.I., and Johnston, S.R. 2005. Endocrine therapy - current benefits and limitations.
Breast Cancer Res. Treat. 93(Suppl. 1):S3-S10.

41. Shou, J., et al. 2004. Mechanisms of tamoxifen resistance: increased estrogen receptor-HER2/neu cross-talk in ER/HER2-positive breast cancer. J. Natl. Cancer Inst. 96:926-935.

42. Jordan, V.C., and Murphy, C.S. 1990. Endocrine pharmacology of antiestrogens as antitumor agents. Endocr. Rev. 11:578-610.

43. Michalides, R., et al. 2002. Cyclin A is a prognostic indicator in early stage breast cancer with and without tamoxifen treatment. Br. J. Cancer. 86:402-408.

44. Dhillon, N.K., and Mudryj, M. 2002. Ectopic expression of cyclin $\mathrm{E}$ in estrogen responsive cells abrogates antiestrogen mediated growth arrest. Oncogene. 21:4626-4634.

45. Naderi, A., et al. 2006. A gene-expression signature to predict survival in breast cancer across independent data sets. Oncogene. doi:10.1038/ sj.onc. 1209920

46. Sorlie, T., et al. 2001. Gene expression patterns of breast carcinomas distinguish tumor subclasses with clinical implications. Proc. Natl. Acad. Sci. U.S. A. 98:10869-10874.

47. Sotiriou, C., et al. 2006. Gene expression profiling in breast cancer: understanding the molecular basis of histologic grade to improve prognosis. J. Natl. Cancer Inst. 98:262-272.

48. van de Vijver, M.J., et al. 2002. A gene-expression signature as a predictor of survival in breast cancer. N. Engl. J. Med. 347:1999-2009.

49. Lee, E.Y., et al. 1988. Inactivation of the retinoblastoma susceptibility gene in human breast cancers. Science. 241:218-221.

50. Loden, M., et al. 2002. The cyclin D1 high and cyclin E high subgroups of breast cancer: separate pathways in tumorogenesis based on pattern of genetic aberrations and inactivation of the $\mathrm{pRb}$ node. Oncogene. 21:4680-4690.

51. Pacilio, C., et al. 1998. Constitutive overexpression of cyclin D1 does not prevent inhibition of hormone-responsive human breast cancer cell growth by antiestrogens. Cancer Res. 58:871-876.

52. Bindels, E.M., Lallemand, F., Balkenende, A., Verwoerd, D., and Michalides, R. 2002. Involvement of $\mathrm{G} 1 / \mathrm{S}$ cyclins in estrogen-independent proliferation of estrogen receptor-positive breast cancer cells. Oncogene. 21:8158-8165.

53. Wang, S., Zhang, B., and Faller, D.V. 2004. BRG1/ $B R M$ and prohibitin are required for growth suppression by estrogen antagonists. EMBO J. 23:2293-2303.

54. Cariou, S., et al. 2000. Down-regulation of p21WAF1/CIP1 or p27Kip1 abrogates antiestrogen-mediated cell cycle arrest in human breast cancer cells. Proc. Natl. Acad. Sci. U. S. A. 97:9042-9046.

55. Wise-Draper, T.M., et al. 2006. Apoptosis inhibition by the human DEK oncoprotein involves interference with p53 functions. Mol. Cell. Biol. 26:7506-7519.

56. Bosco, E.E., et al. 2004. RB signaling prevents replication-dependent DNA double-strand breaks following genotoxic insult. Nucleic Acids Res. 32:25-34.

57. Markey, M.P., et al. 2002. Unbiased analysis of RB-mediated transcriptional repression identifies novel targets and distinctions from E2F action. Cancer Res. 62:6587-6597. 